\title{
Genome-Wide Transcriptome Analysis of Cadmium Stress in Rice
}

\author{
Youko Oono, Takayuki Yazawa, Hiroyuki Kanamori, Harumi Sasaki, Satomi Mori, \\ Hirokazu Handa, and Takashi Matsumoto
}

Agrogenomics Research Center, National Institute of Agrobiological Sciences, Tsukuba, Ibaraki 305-8602, Japan

Correspondence should be addressed to Youko Oono; yoono@affrc.go.jp

Received 26 November 2015; Revised 26 January 2016; Accepted 28 January 2016

Academic Editor: Yan-Bo Hu

Copyright (C) 2016 Youko Oono et al. This is an open access article distributed under the Creative Commons Attribution License, which permits unrestricted use, distribution, and reproduction in any medium, provided the original work is properly cited.

Rice growth is severely affected by toxic concentrations of the nonessential heavy metal cadmium (Cd). To elucidate the molecular basis of the response to Cd stress, we performed mRNA sequencing of rice following our previous study on exposure to high concentrations of $\mathrm{Cd}$ (Oono et al., 2014). In this study, rice plants were hydroponically treated with low concentrations of Cd and approximately 211 million sequence reads were mapped onto the IRGSP-1.0 reference rice genome sequence. Many genes, including some identified under high Cd concentration exposure in our previous study, were found to be responsive to low Cd exposure, with an average of about 11,000 transcripts from each condition. However, genes expressed constitutively across the developmental course responded only slightly to low Cd concentrations, in contrast to their clear response to high Cd concentration, which causes fatal damage to rice seedlings according to phenotypic changes. The expression of metal ion transporter genes tended to correlate with Cd concentration, suggesting the potential of the RNA-Seq strategy to reveal novel Cd-responsive transporters by analyzing gene expression under different $\mathrm{Cd}$ concentrations. This study could help to develop novel strategies for improving tolerance to Cd exposure in rice and other cereal crops.

\section{Introduction}

Cadmium $(\mathrm{Cd})$ is a widespread heavy metal pollutant that is highly toxic to living cells. Accumulation of the nonessential metal Cd in plants is a major agricultural problem. Specifically, Cd is absorbed by the roots from the soil and transported to the shoot, negatively affecting nutrient uptake and homeostasis in plants, even in very small amounts. Many agricultural soils have become contaminated with Cd through the use of phosphate fertilizers, sludge, and irrigation water containing $\mathrm{Cd}$. Cd exposure inhibits root and shoot growth and ultimately reduces yield. Furthermore, $\mathrm{Cd}$ accumulation in the edible parts of plants such as seed grains places humans at a risk because of its highly toxic effects on human health. Reducing the Cd concentration in plants below the maximum level indicated by the Codex Alimentarius Commission of FAO/WHO [1] is necessary to avoid negative impacts on human health. Thus, it is important to study the mechanisms of plant responses and defenses to Cd exposure to overcome this problem.
Cd causes oxidative stress and generates reactive oxygen species, which can cause damage in various ways such as reacting with DNA causing mutation, modifying protein side chains, and destroying phospholipids [2]. Various biochemical and physiological processes associated with defense systems are active in plants under Cd exposure. Many genes such as glutathione S-transferase (GST) for detoxification and cysteine-rich metallothioneins (MT) for defense against $\mathrm{Cd}$ toxicity respond to $\mathrm{Cd}$ stress in plants and might confer $\mathrm{Cd}$ tolerance in rice. Transporters with heavy metal binding domains are key factors for root uptake of $\mathrm{Cd}$ from soil and efflux pumping of $\mathrm{Cd}$ at the plasma membrane; however, the manner in which these genes respond to low Cd concentrations has not been well investigated in rice.

In a previous study, we investigated the gene expression of rice plants (Oryza sativa L. cv. Nipponbare) under a high $\mathrm{Cd}$ concentration using the RNA-Seq platform. A clear and detailed view of the transcriptomic changes triggered by $\mathrm{Cd}$ exposure is important to understand the gene expression network of the basal response to Cd stress. This could not be 
obtained from past studies using the microarray platform, but RNA-Seq can accurately quantify gene expression levels over a broad dynamic range with high resolution and sensitivity [3]. We found that drought stress signaling pathways were activated under $\mathrm{Cd}$ exposure through the responses of many drought-related genes [4]. Thus, the recently elucidated scaffolding mechanisms for Cd signaling pathways are complex but of great importance. In this study, we performed rice transcriptome analysis under different low $\mathrm{Cd}$ concentrations using the RNA-Seq platform to deepen our understanding of $\mathrm{Cd}$ responses.

\section{Materials and Methods}

2.1. Sample Preparation. Rice (Oryza sativa ssp. japonica cv. Nipponbare) seeds were germinated and grown by hydroponic culture in Yoshida's solution $\left[1.425 \mathrm{mM} \mathrm{NH}_{4} \mathrm{NO}_{3}\right.$, $0.323 \mathrm{mM} \mathrm{NaH}_{2} \mathrm{PO}_{4}, 0.513 \mathrm{mM} \mathrm{K} \mathrm{SO}_{4}, 0.998 \mathrm{mM} \mathrm{CaCl}$, $1.643 \mathrm{mM} \mathrm{MgSO}_{4}, 0.009 \mathrm{mM} \mathrm{MnCl}, 0.075 \mathrm{mM}\left(\mathrm{NH}_{4}\right)_{6}$ $\mathrm{Mo}_{7} \mathrm{O}_{24}, 0.019 \mathrm{mM} \mathrm{H} \mathrm{BO}_{3}, 0.155 \mathrm{mM} \mathrm{CuSO}, 0.036 \mathrm{mM}$ $\mathrm{FeCl}_{3}, 0.070 \mathrm{mM}$ citric acid, and $0.152 \mathrm{mM} \mathrm{ZnSO}_{4}$ ] [5]. After 10 days, seedlings of uniform size and growth were subjected to $\mathrm{Cd}$ stress treatment by transferring them to a similar medium with $0.2,1$, or $50 \mu \mathrm{M} \mathrm{Cd}$. These values were chosen based on a report that the total dissolved Cd in 64 fields with Cd-contaminated soils ranged from 0.03 to $182 \mu \mathrm{g} / \mathrm{L}$ [6] in previous experiences. The plants were maintained under Cd stress conditions for $14 \mathrm{~d}$. Root and shoot samples were collected at approximately 9:00 AM, frozen in liquid nitrogen, and stored at $-80^{\circ} \mathrm{C}$ until subsequent analyses. Total RNA was extracted from both root and shoot samples using an RNeasy Plant Kit (Qiagen, Hilden, Germany) according to the manufacturer's instructions. Construction of 34 cDNA libraries ( 2 tissues, 4 conditions, 2 treatments, and 2-3 replicates) from total RNA using a TruSeq RNA sample preparation kit and sequencing with the Illumina Genome Analyzer IIx (Illumina Inc., San Diego, CA, USA) was performed according to the manufacturer's protocols as a part of establishing TENOR (Transcriptome Encyclopedia of Rice, http://tenor.dna.affrc.go.jp/) [7]. The resulting RNASeq data were deposited in the DDBJ Sequence Read Archive (Accession number DRA000959).

2.2. Identification of Differentially Expressed Transcripts. The biological replicates (2-3) for each set of conditions were highly correlated (coefficient $>0.95$ ), so reads from the same treatment were merged for subsequent analysis. Trimming of Illumina adaptor sequences and low-quality bases $(Q<20)$ by Cutadapt [8] and mapping of preprocessed reads to the IRGSP-1.0 genome assembly (http://rapdb.dna.affrc.go.jp/) were performed as described previously [9]. To estimate the expression levels of each transcript, all preprocessed reads were mapped to the IRGSP-1.0 genome assembly by Bowtie with default parameters [10]. The expression level for each transcript was calculated as the RPKM- (Reads per Kilobase Exon Model per million mapped reads-) derived read count [11] based on the number of uniquely mapped reads that overlapped with exonic regions. A G-test was performed to detect differentially expressed transcripts in the control and $\mathrm{Cd}$ treatments based on the statistical null hypothesis that the proportions of mapped reads to the transcripts were the same between the two conditions. A false discovery rate (FDR < 0.01) was used in multiple-hypothesis testing to correct for multiple comparisons. When calculating fold changes, 1 was added to avoid division by 0 .

\subsection{Hierarchical Clustering and Gene Ontology Enrichment} Analysis. The Cd-responsive transcripts in root and shoot were used for hierarchical clustering analysis. We used the heatmap. 2 in the R package gplots (version 2.11.0) to perform clustering analyses of transcripts. The $Z$ scores were used to compare significant changes in gene expression. A Gene Ontology (GO) term was assigned to each transcript based on the GO annotations for biological process, molecular function, and cellular component in RAP-DB. GO enrichment was evaluated by Fisher's exact test with a FDR threshold of $5 \%$ for responsive transcripts in the biological process category of each cluster. The results were plotted as $-\log 10$ of FDR values in a heatmap.

2.4. $q R T-P C R$ Analysis. The expression of Cd upregulated genes in root sample was confirmed by qRT-PCR analysis. Rice seeds were germinated and grown in water in a growth chamber. After 10 days, the seedlings were subjected to different stress treatments by transferring them to water containing different reagents. RNA was extracted from them and the cDNA was synthesized according to the manufacturer's protocol and it is used for the further analysis as described previously [4]. The resulting cDNA was used for PCR amplification in the LightCycler 480 system (Roche, Basel, Switzerland) with each primer set (Os04g0600300: $5^{\prime}$-GGCGCTCTGAGAATCATCAC-3', 5'-CATTCGGGAGCTCATCTCG3', Os01g0692100: $5^{\prime}$-ATTCACGAGTCCGCGATG-3', $5^{\prime}$ CTCTCACCCGGATCACCC-3', Os12g0570700: $5^{\prime}$-GCACTCATCTCAAGCTTTTC- $3^{\prime}, 5^{\prime}$-GCAAGACATCTTCTTGG-3', Os12g0571000: 5'-ATTTCCTGAAGAGTTAAA-3', $5^{\prime}$-TTCCGCAGCCGCAGCTTA- $3^{\prime}$ ). The detection threshold cycle for each reaction was normalized using Ubiquitinl primers $\left(5^{\prime}\right.$-CCAGGACAAGATGATCTGCC- $3^{\prime}, 5^{\prime}$ AAGAAGCTGAAGCATCCAGC-3').

\section{Results and Discussion}

3.1. Low Cd Concentration Exposure of Rice Plants and Growth Retardation during the Treatment. We used rice plants grown in hydroponic culture, which enabled us to control the Cd exposure easily. High Cd concentration exposure has been previously shown to elicit robust physiological responses and gene expression as acute toxic responses in rice seedlings [1214]. Growth retardation of the shoot was slightly visible after $1 \mathrm{~d}$ (data not shown), the leaves turned yellow and the leaf tips of the seedlings began to wilt after $4 \mathrm{~d}$, and all leaf blades were curled completely and the seedlings were wilting after $10 \mathrm{~d}$ under high Cd concentration $(50 \mu \mathrm{M})$ exposure (Figure 1). While no visible symptoms were observed in shoots under low $\mathrm{Cd}$ concentration exposure $(0.2$ and $1 \mu \mathrm{M} \mathrm{Cd})$ after 

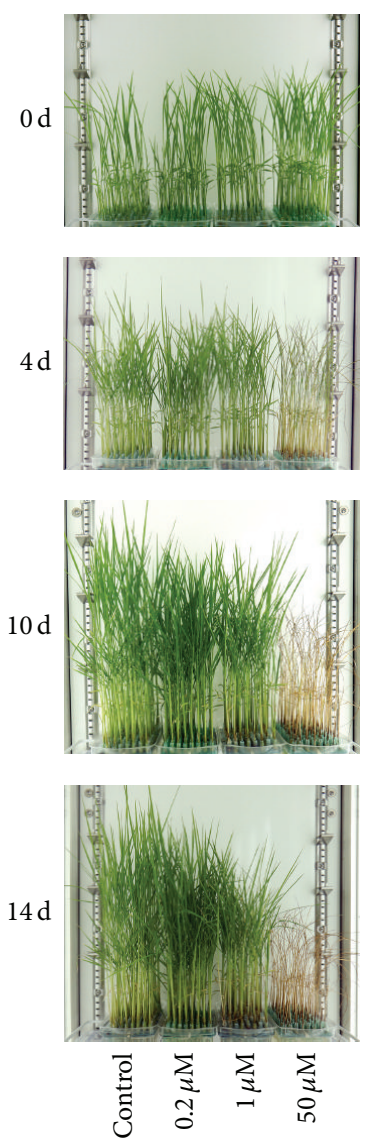

Figure 1: Phenotypic changes in rice plants grown in culture medium with low concentrations of $\mathrm{Cd}(0.2,1 \mu \mathrm{M})$ and a high concentration of $\mathrm{Cd}(50 \mu \mathrm{M})$ from 0 to $14 \mathrm{~d}$.

$1 \mathrm{~d}$, growth retardation occurred gradually compared with the control, with symptoms starting to appear after $7 \mathrm{~d}$. Plants in the same growth chamber exposed to different Cd concentrations showed clear growth differences after $10 \mathrm{~d}$ (Figure 1). Even after $28 \mathrm{~d}$, the seedlings under low $\mathrm{Cd}$ concentration exposure did not show yellow leaves or wilting (data not shown). These results suggested that high $\mathrm{Cd}$ concentration exposure causes fatal damage to plants while low Cd concentrations lead to growth retardation (Figure 1), which is supported by the fact that plant detoxification processes are insufficient to cope with this toxic metal beyond a $10 \mu \mathrm{M}$ dose [15].

3.2. Gene Expression Profiles under Low Cd Concentration Exposure in Rice. We next analyzed the transcriptome profiles of the response to Cd exposure using RNA-Seq during plant growth, at 1,4 , and $10 \mathrm{~d}$ after $\mathrm{Cd}$ treatment, and before treatment $(0 \mathrm{~d})$. For each set of conditions, an average of approximately 15.1 million (92.2\%) quality-evaluated reads (total 211 million) were mapped to the rice genome sequence and used for further analysis (Table S1 in Supplementary Material available online at http://dx.doi.org/10.1155/2016/9739505). The number of upregulated transcripts ranged from 4,529 to 6,515 , whereas

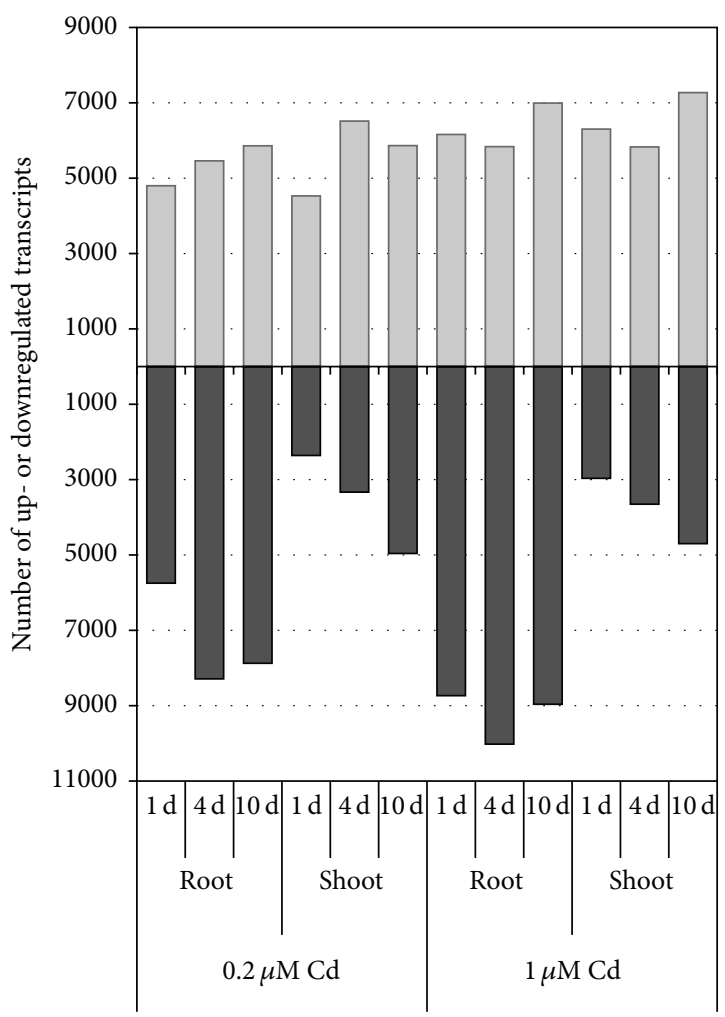

Figure 2: Distribution of upregulated and downregulated transcripts in roots and shoots in response to Cd exposure. RPKM fold changes at 1,4 , and $10 \mathrm{~d}$ were calculated for Cd-treated samples compared with nontreated samples $(0 \mathrm{~d})$. The total numbers of upregulated (upper) and downregulated (lower) transcripts in roots and shoots identified by RNA-Seq were determined by G-tests (FDR $<0.01)$ at each stress time point $(1,4$, and $10 \mathrm{~d}$ ) under $0.2 \mu \mathrm{M}$ (left) and $1 \mu \mathrm{M}$ (right) Cd exposure. The $x$-axis shows the time course and the $y$-axis shows the number of transcripts.

the number of downregulated transcripts ranged from 2,359 to 8,734 under $0.2 \mu \mathrm{M}$ Cd (Figure 2). Twelve transcripts including GST, MT, and DREB (drought responsive element binding protein) $1 \mathrm{E}$ were upregulated more than 20 -fold among the upregulated transcripts in roots at $0.2 \mu \mathrm{M} \mathrm{Cd}$. The number of upregulated transcripts ranged from 5,830 to 7,271 whereas the number of downregulated transcripts ranged from 2,965 to 10,020 under $1 \mu \mathrm{M} \mathrm{Cd}$ (Figure 2). Fifty-one transcripts including GST, MT, Prx (peroxidase), and heat shock proteins were upregulated more than 20fold among the upregulated transcripts in roots at $1 \mu \mathrm{M}$ Cd (Table 1). Induction of detoxification enzymes against oxidation stress such as GST and Prx under Cd exposure might be associated with the defense system that confers $\mathrm{Cd}$ tolerance to plants [16-18] even at low $\mathrm{Cd}$ concentrations. The cysteine-rich MT might function as a ligand for chelation of metal ions to defend against Cd toxicity [19]. The DREB/Crepeat binding factor $(\mathrm{CBF})$ specifically interacts with the DRE/CRT cis-acting element and controls the expression of many stress-inducible genes in plants [20]. The activation of gene expression in several drought stress signal pathways under Cd exposure has been reported [4]. Five heat shock 
TABLE 1: Cadmium-upregulated transcripts identified in roots by RNA-Seq analysis.

\begin{tabular}{|c|c|c|c|c|c|c|c|}
\hline \multirow{3}{*}{ Transcript } & \multirow{3}{*}{ Description } & \multicolumn{6}{|c|}{ Fold change } \\
\hline & & \multicolumn{3}{|c|}{ Root } & \multicolumn{3}{|c|}{ Shoot } \\
\hline & & $1 \mathrm{~d}$ & $4 \mathrm{~d}$ & $10 \mathrm{~d}$ & $1 \mathrm{~d}$ & $4 \mathrm{~d}$ & $10 \mathrm{~d}$ \\
\hline \multicolumn{8}{|l|}{$0.2 \mu \mathrm{M} \mathrm{Cd}$} \\
\hline Os10t0527400-01 & Tau class GST protein 3 & 27.8 & 21.4 & 27.5 & 1.2 & 2.0 & 1.7 \\
\hline Os03t0283000-00 & In2-1 protein & 27.5 & 2.8 & 1.0 & 1.3 & 1.1 & 1.5 \\
\hline Os08t0156000-01 & Conserved hypothetical protein & 26.4 & 21.4 & 25.3 & 1.3 & 1.6 & 1.7 \\
\hline Os01t0627967-00 & Hypothetical protein & 26.1 & 16.5 & 24.1 & 1.5 & 1.9 & 1.4 \\
\hline Os04t0178300-02 & Syn-copalyl diphosphate synthase & 20.1 & 8.0 & 20.3 & 0.6 & 4.2 & 1.4 \\
\hline Os04t0301500-01 & $\begin{array}{l}\text { HLH (helix-loop-helix) DNA-binding } \\
\text { domain containing protein }\end{array}$ & 0.4 & 33.1 & 0.5 & 1.0 & 47.5 & 9.2 \\
\hline Os02t0676800-01 & $\begin{array}{l}\text { DREB1E (drought responsive element } \\
\text { binding protein } 1 \mathrm{E} \text { ) }\end{array}$ & 0.9 & 28.7 & 0.9 & 1.2 & 10.9 & 2.0 \\
\hline Os02t0179200-01 & $\begin{array}{l}\text { Glutamine amidotransferase class-I domain } \\
\text { containing protein }\end{array}$ & 0.8 & 28.1 & 1.7 & 0.9 & 3.2 & 1.1 \\
\hline Os12t0154800-00 & $\begin{array}{l}\text { RmlC-like jelly roll fold domain containing } \\
\text { protein }\end{array}$ & 4.0 & 21.4 & 5.7 & 1.0 & 1.4 & 1.2 \\
\hline Os12t0570700-01 & MT (metallothionein)-like protein type 1 & 18.6 & 20.3 & 15.8 & 0.9 & 1.0 & 0.9 \\
\hline Os03t0836800-01 & IAA-amino acid hydrolase 1 & 4.3 & 6.5 & 33.6 & 1.0 & 1.0 & 1.0 \\
\hline Os10t0333700-00 & $\begin{array}{c}\text { Plant disease resistance response protein } \\
\text { domain containing protein }\end{array}$ & 9.7 & 6.0 & 21.6 & 1.0 & 1.0 & 1.0 \\
\hline \multicolumn{8}{|l|}{$1 \mu \mathrm{M} \mathrm{Cd}$} \\
\hline Os04t0178300-02 & Syn-copalyl diphosphate synthase & 122.0 & 32.1 & 25.5 & 0.5 & 1.0 & 3.6 \\
\hline Os04t0178300-01 & $\begin{array}{c}\text { Isoform } 3 \text { of Syn-copalyl diphosphate } \\
\text { synthase }\end{array}$ & 109.8 & 27.8 & 21.5 & 0.5 & 0.9 & 3.1 \\
\hline Os04t0178400-01 & Cytochrome P450 CYP99A1 & 69.8 & 21.1 & 16.0 & 0.8 & 1.0 & 2.8 \\
\hline Os03t0267000-00 & Heat shock protein 180 & 57.5 & 7.7 & 10.9 & 1.2 & 0.7 & 0.7 \\
\hline Os03t0266900-01 & Heat shock protein 173 & 47.0 & 4.9 & 5.3 & 1.0 & 0.4 & 0.6 \\
\hline Os01t0136200-01 & Heat shock protein 1 & 43.7 & 3.9 & 1.3 & 1.0 & 1.0 & 1.0 \\
\hline Os07t0190000-01 & $\begin{array}{l}\text { 1-Deoxy-D-xylulose } 5 \text {-phosphate synthase } 2 \\
\text { precursor }\end{array}$ & 42.4 & 11.5 & 8.6 & 0.7 & 1.1 & 3.9 \\
\hline Os07t0127500-01 & PR-1a pathogenesis related protein precursor & 40.0 & 5.6 & 5.0 & 0.8 & 0.8 & 2.1 \\
\hline Os07t0154100-01 & Viviparous-14 & 38.8 & 5.2 & 1.5 & 1.1 & 1.4 & 2.3 \\
\hline Os07t0154201-00 & Hypothetical gene & 37.7 & 4.7 & 1.3 & 1.0 & 1.3 & 2.1 \\
\hline Os12t0555200-01 & Probenazole-inducible protein PBZ1 & 37.7 & 13.5 & 10.9 & 0.3 & 0.5 & 2.2 \\
\hline Os06t0586000-01 & Conserved hypothetical protein & 37.6 & 9.3 & 6.5 & 0.6 & 0.9 & 1.4 \\
\hline Os10t0527400-01 & Tau class GST protein 3 & 34.3 & 18.0 & 32.4 & 1.1 & 1.4 & 2.0 \\
\hline Os12t0555000-01 & Probenazole-inducible protein PBZ1 & 33.2 & 13.5 & 11.0 & 0.6 & 0.7 & 2.5 \\
\hline Os03t0277700-01 & $\begin{array}{l}\text { Protein of unknown function DUF26 } \\
\text { domain containing protein }\end{array}$ & 32.8 & 7.6 & 3.4 & 1.0 & 0.6 & 1.0 \\
\hline Os11t0687100-01 & von Willebrand factor (type A domain) & 32.5 & 4.1 & 13.8 & 0.7 & 0.7 & 2.3 \\
\hline Os05t0211700-00 & - & 28.8 & 1.4 & 1.2 & 1.0 & 1.0 & 1.0 \\
\hline Os06t0662550-01 & Conserved hypothetical protein & 28.5 & 7.8 & 8.8 & 0.8 & 0.8 & 1.6 \\
\hline Os01t0944100-02 & Conserved hypothetical protein & 28.4 & 6.3 & 9.8 & 0.5 & 0.6 & 1.7 \\
\hline Os06t0568600-01 & Ent-kaurene oxidase 1 & 27.1 & 28.1 & 11.0 & 0.6 & 1.4 & 4.7 \\
\hline Os12t0418600-01 & Hypothetical conserved gene & 26.7 & 2.0 & 1.3 & 1.0 & 1.0 & 1.0 \\
\hline Os12t0258700-01 & Cupredoxin domain containing protein & 26.2 & 14.7 & 10.6 & 0.7 & 1.1 & 7.1 \\
\hline Os01t0615100-01 & Substilin/chymotrypsin-like inhibitor & 25.6 & 9.5 & 7.9 & 0.7 & 1.0 & 1.8 \\
\hline Os04t0107900-02 & Heat shock protein $81-1$ & 25.6 & 2.5 & 1.6 & 1.0 & 1.0 & 0.9 \\
\hline
\end{tabular}


TABLE 1: Continued.

\begin{tabular}{|c|c|c|c|c|c|c|c|}
\hline \multirow{3}{*}{ Transcript } & \multirow{3}{*}{ Description } & \multicolumn{6}{|c|}{ Fold change } \\
\hline & & \multicolumn{3}{|c|}{ Root } & \multicolumn{3}{|c|}{ Shoot } \\
\hline & & $1 \mathrm{~d}$ & $4 \mathrm{~d}$ & $10 \mathrm{~d}$ & $1 \mathrm{~d}$ & $4 \mathrm{~d}$ & $10 \mathrm{~d}$ \\
\hline Os09t0493000-01 & Conserved hypothetical protein & 25.3 & 2.6 & 1.8 & 0.9 & 1.2 & 0.9 \\
\hline Os01t0627967-00 & Hypothetical protein & 25.3 & 19.5 & 21.6 & 1.3 & 1.8 & 1.4 \\
\hline Os01t0944100-03 & Conserved hypothetical protein & 25.2 & 4.6 & 6.3 & 0.6 & 0.6 & 1.8 \\
\hline Os04t0180400-01 & Cytochrome P450 99A2 & 24.4 & 4.3 & 6.0 & 0.5 & 0.5 & 3.1 \\
\hline Os04t0108101-00 & Hypothetical protein & 24.4 & 2.3 & 1.4 & 1.0 & 1.0 & 1.0 \\
\hline Os02t0269600-00 & Subtilase & 22.6 & 7.8 & 4.1 & 0.3 & 1.2 & 6.0 \\
\hline Os01t0136000-00 & Heat shock protein 175 & 22.5 & 3.1 & 1.2 & 1.0 & 1.4 & 1.2 \\
\hline Os04t0180500-00 & Hypothetical protein & 22.2 & 4.0 & 5.4 & 0.5 & 0.6 & 3.1 \\
\hline Os01t0946600-01 & Conserved hypothetical protein & 21.8 & 16.6 & 8.0 & 0.7 & 0.7 & 0.8 \\
\hline Os09t0255400-02 & Indole-3-glycerol phosphate synthase & 21.4 & 5.1 & 3.8 & 0.7 & 0.9 & 2.3 \\
\hline Os01t0348900-01 & SalT gene product & 21.2 & 6.5 & 8.9 & 0.1 & 0.1 & 0.2 \\
\hline Os12t0491800-01 & Ent-kaurene synthase $1 \mathrm{~A}$ & 21.1 & 1.5 & 1.7 & 0.4 & 0.8 & 5.5 \\
\hline Os01t0132000-01 & Wound-induced protease inhibitor & 21.0 & 8.8 & 11.6 & 1.6 & 0.5 & 0.2 \\
\hline Os11t0592200-01 & Chitin-binding allergen Bra r 2 & 20.7 & 3.4 & 2.8 & 0.7 & 0.5 & 1.6 \\
\hline Os01t0963000-01 & Prx (Peroxidase) BP 1 precursor & 20.6 & 3.8 & 4.4 & 0.7 & 1.1 & 1.3 \\
\hline Os08t0189600-01 & Oryza sativa germin-like protein $8-7$ & 20.6 & 11.5 & 6.7 & 2.1 & 1.5 & 0.8 \\
\hline Os07t0496250-01 & Expansin-like B1 & 20.5 & 2.2 & 2.2 & 1.5 & 1.2 & 4.5 \\
\hline Os01t0963000-04 & Prx (Peroxidase) BP 1 precursor & 20.3 & 3.7 & 4.4 & 0.7 & 1.1 & 1.3 \\
\hline Os09t0255400-01 & Indole-3-glycerol phosphate synthase & 20.2 & 5.2 & 3.7 & 0.7 & 0.9 & 2.3 \\
\hline Os11t0601950-01 & cDNA clone:002-114-B06 & 20.0 & 1.7 & 1.9 & 0.7 & 1.0 & 1.1 \\
\hline Os03t0129400-01 & Hypothetical protein & 10.3 & 27.1 & 17.6 & 1.0 & 1.9 & 3.6 \\
\hline Os01t0322700-01 & Nonprotein coding transcript & 12.2 & 25.5 & 15.7 & 0.9 & 1.3 & 2.5 \\
\hline Os03t0129400-02 & $\begin{array}{l}\text { EST AU078206 corresponds to a region of } \\
\text { the predicted gene }\end{array}$ & 9.4 & 24.3 & 16.3 & 1.1 & 1.4 & 2.8 \\
\hline Os12t0570700-01 & MT (metallothionein)-like protein type 1 & 16.7 & 21.2 & 17.7 & 0.8 & 0.8 & 3.1 \\
\hline Os12t0571000-01 & MT (metallothionein)-like protein type 1 & 13.9 & 20.0 & 13.0 & 0.9 & 1.0 & 3.6 \\
\hline Os08t0156000-01 & Conserved hypothetical protein & 15.4 & 17.9 & 26.0 & 1.1 & 1.5 & 1.6 \\
\hline Os03t0836800-01 & IAA-amino acid hydrolase 1 & 0.7 & 4.0 & 23.7 & 1.0 & 1.0 & 1.0 \\
\hline
\end{tabular}

Reads were mapped to the rice genome and responsive genes were identified by $G$-tests. Transcripts upregulated more than 20 -fold in one or more treatments/time points in roots are shown. Transcripts in bold were upregulated under both 1 and $0.2 \mu \mathrm{M}$ Cd exposure.

proteins (Hsps) were strongly upregulated in roots under $1 \mu \mathrm{M}$ $\mathrm{Cd}$, with the greatest relative expression at $1 \mathrm{~d}$ (Table 1). These genes may contribute to cellular homeostasis by protecting macromolecules such as enzymes, protein complexes, and membranes under Cd exposure. This result suggested that the roots of hydroponically cultured rice might be affected more directly and earlier by $\mathrm{Cd}$ exposure. There was a difference between the low $\mathrm{Cd}$ concentrations in that no Hsps were strongly upregulated in roots at $0.2 \mu \mathrm{M}$ Cd (Table 1), suggesting that the effect of this condition might be small or show time lag. In shoots, 15 and 11 transcripts were upregulated more than 20-fold among the upregulated transcripts under 0.2 and $1 \mu \mathrm{M} \mathrm{Cd}$, respectively (Table S2). Nine transcripts including Nramp1 (natural resistance-associated macrophage protein) were upregulated under both 0.2 and $1 \mu \mathrm{M} \mathrm{Cd}$ (Table S2). In Arabidopsis, Nramp1 localizes to the plasma membrane and functions as a high-affinity transporter for manganese (Mn) uptake [21], while OsNramp5 uptakes Mn and Cd [22]. Transporters with heavy metal binding domains are often capable of transporting several metals, such as Fe, $\mathrm{Zn}, \mathrm{Mn}$, and $\mathrm{Cd}$, because of their low substrate specificity [23-26]. We found that upregulation of a HLH DNA-binding domain containing transcription factor (Os04g0301500) in both roots and shoots peaked at $4 \mathrm{~d}$ under $0.2 \mu \mathrm{M} \mathrm{Cd}$; this protein may function as a regulatory factor under $\mathrm{Cd}$ exposure (Table 1, Table S2). The number of downregulated transcripts in roots peaked at $4 \mathrm{~d}$ after $\mathrm{Cd}$ exposure, while the number in shoots gradually increased under low $\mathrm{Cd}$ concentration exposure (Figure 2). A few dozen transcripts were downregulated less than 0.05 -fold among the downregulated transcripts in roots and shoots under Cd exposure (Table S2). Therefore, a small part of transcripts were strongly 
up- or downregulated among several thousand responsive transcripts under low $\mathrm{Cd}$ concentration exposure. Largescale changes in gene expression occurred in rice under $\mathrm{Cd}$ exposure, even at low concentrations, possibly because $\mathrm{Cd}$ is a nonessential metal for the plant.

To obtain a functional annotation of responsive transcripts under Cd exposure, we used GO biological process categories. The responsive transcripts in shoot and root were clustered into several groups based on their expression patterns. GO enrichment analysis was performed using clustered transcripts assigned by GO terms in RAP-DB (The Rice Annotation Project Database [http://rapdb.dna.affrc.go.jp/]) (Supplementary Figure S1). Enriched GO terms significantly in each cluster may represent the functional categories in rice under $\mathrm{Cd}$ exposure. Enriched GO terms of gradually upregulated transcripts under $\mathrm{Cd}$ exposure include metal ion transport (GO:0030001) (cluster 3 in root under $0.2 \mu \mathrm{M}$ $\mathrm{Cd}$, cluster 4 in root under $1 \mu \mathrm{M} \mathrm{Cd}$ ), which may function in Cd transport. Response to oxidative stress (GO:0006979) and responsive to oxidative stress (GO:0006979) were also included in cluster 3 and cluster 4, respectively. This suggested that they might function in defense against $\mathrm{Cd}$. Enriched GO terms of gradually downregulated transcripts under Cd exposure include translation (GO:0006412), translation elongation (GO:0006414), DNA replication (GO:0006260), and DNA repair (GO:0006281) (cluster 1 in root under $0.2 \mu \mathrm{M}$ $\mathrm{Cd}$, cluster 2 in root under $1 \mu \mathrm{M} \mathrm{Cd})$. Photosynthesis, light harvesting (GO:0009765), and photosynthesis (GO:0015979) were also included in both clusters. These may function in plant growth. Thus, these correspond to the observed changes in phenotype (Figure 1), which clearly validated the RNA-Seq expression profiling data obtained from rice tissue under $\mathrm{Cd}$ stress condition. However, the pattern of gene expression is quite complex and would require more detailed analysis.

3.3. Constitutively Expressed Genes Responded Differently under Low Cd Concentration to High Cd Concentration. As many genes responded to both low and high Cd concentrations [4], we assessed the effect of the stress degree on rice seedlings through the expression of constitutively expressed genes. We investigated the expression of 18 genes annotated by the RAP that were expressed constitutively in 39 tissues collected throughout the life cycle of the rice plant from two varieties according to 190 Affymetrix GeneChip Rice Genome Arrays, in addition to four genes annotated by the RAP that have frequently been used as internal controls in expression analyses [27]. The results showed that the expression of more than half of them fluctuated drastically $(>2$ or $<2)$ in roots or shoots after $1 \mathrm{~d}$ of high $\mathrm{Cd}$ concentration exposure (Figure 3). This drastic response may be partly because RNA-Seq can accurately quantify gene expression levels over a broad dynamic range with high resolution and sensitivity $[10,28,29]$. However, our results suggest that their expression is greatly affected by strong stress, even though they are expressed constitutively across the developmental course. Note that a high $\mathrm{Cd}$ concentration can cause fatal damage to rice seedlings, such as by affecting homeostasis, which corresponds to the observed changes in phenotype (Figures 1 and 3).
3.4. Comparative Gene Expression Analysis between Low and High Cd Concentrations Reveals Novel Cd-Responsive Transporters. We investigated the expression of metal transporter genes containing metal ion binding Pfam domains [PF01554 (MatE), PF08370 (PDR_assoc), PF01545 (Cation_efflux), PF02535 (Zip), PF00403 (HMA), and PF01566 (Nramp)] that may function in $\mathrm{Cd}$ transport under $\mathrm{Cd}$ exposure. The expression of 183 transport transcripts was compared between low and high $\mathrm{Cd}$ concentration treatments in roots and shoots at $1 \mathrm{~d}$, because Cd uptake from the hydroponic culture and efflux pumping are initial responses to $\mathrm{Cd}$ exposure (Figure 4, Table S3). The transcripts tended to be more responsive in roots and shoots under higher $\mathrm{Cd}$ concentration exposure. This result indicated the potential of the RNA-Seq strategy to reveal novel Cd-responsive transporters by analyzing gene expression under exposure to different $\mathrm{Cd}$ concentrations. The responsive transcripts might function in roots at the early stage of $\mathrm{Cd}$ exposure. No transcripts were upregulated more than 3-fold in shoots under low Cd exposure (Figure 4, Table S3), suggesting that the effect takes more time to appear in shoots. Os03g0667500 (Zip, root) encoding iron-regulated transporter 1 (IRT1) was upregulated more than 5-fold under low $\mathrm{Cd}$ concentrations but responded only slightly under the high $\mathrm{Cd}$ concentration. IRT1s often transport Cd because of their low substrate specificity [24-26, 30]. Os02g0585200 (HMA, root), Os03g0152000 (HMA, root), Os0g0584800 (HMA, root), Os01g0609900 (PDR_assoc, shoot), and Os01g0609300 (PDR_assoc, shoot) showed the highest (32-fold) upregulation under high $\mathrm{Cd}$ concentration exposure and responded only slightly to low $\mathrm{Cd}$ concentrations (Table S3). The balance between $\mathrm{Cd}$ and various other metal ions in the hydroponic culture might affect the expression of these genes, because specific systems for transporting Cd may have not developed in rice as it is a nonessential metal. The effects of other ions on the expression of transporters [4] and responsive genes associated with defense systems against Cd (Supplementary Figure S2) have been indicated.

\section{Conclusions}

We generated gene expression profiles for rice seedlings grown under low $\mathrm{Cd}$ concentrations. Phenotypic observations and constitutive gene expression indicated that low $\mathrm{Cd}$ concentrations cause growth retardation but are far from being fatal in rice. Several genes associated with defense systems were strongly upregulated; the expression of metal ion transporter genes tended to correlate with $\mathrm{Cd}$ concentration and GO enrichment analysis of the clustered genes based on their expression patterns, suggesting that our transcriptome profiles reflect responses to Cd in rice. Our data also suggest that it could be dangerous to eat plants that do not show specific Cd pollution symptoms growing in soil contaminated by small amounts of $\mathrm{Cd}$. Establishing the exact composition and organization of the transcriptional network underlying the response to Cd exposure will provide a robust tool for improving crops in the future, for example, by creating low Cd uptake plants. 


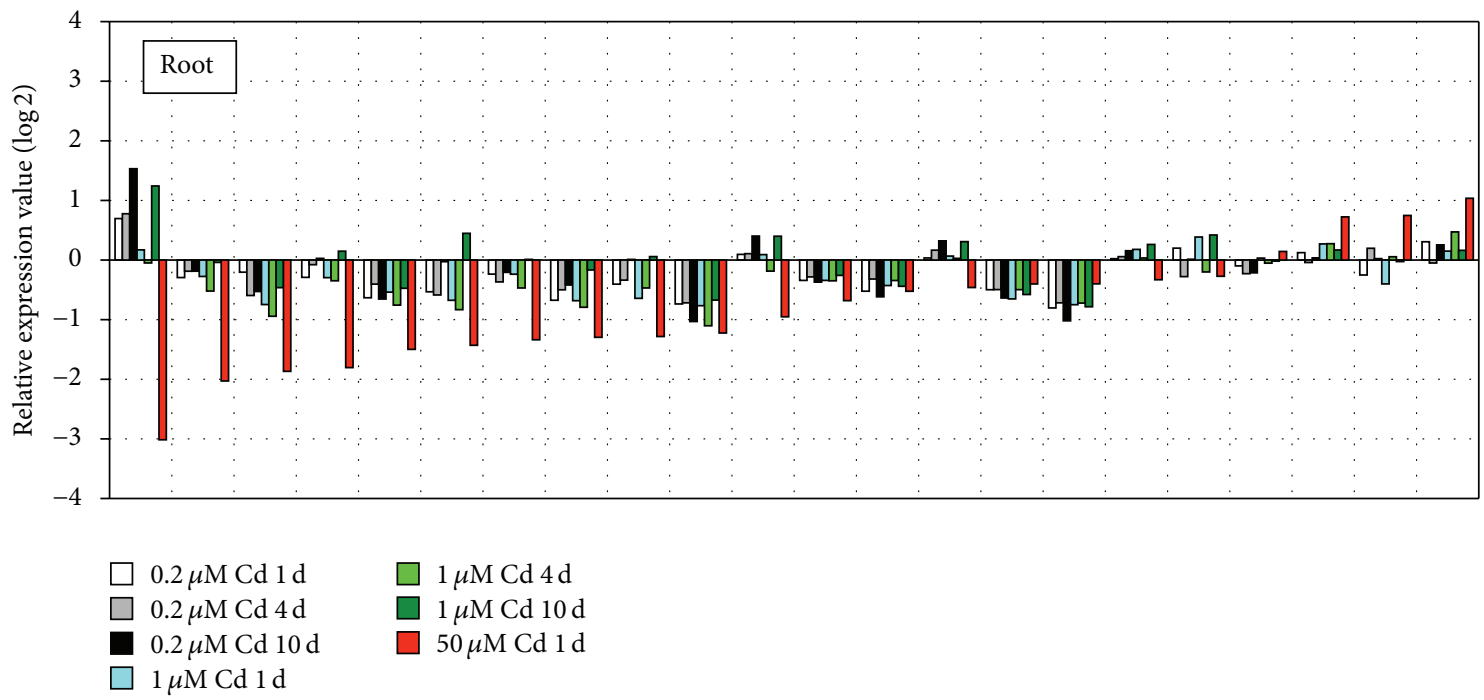

(a)

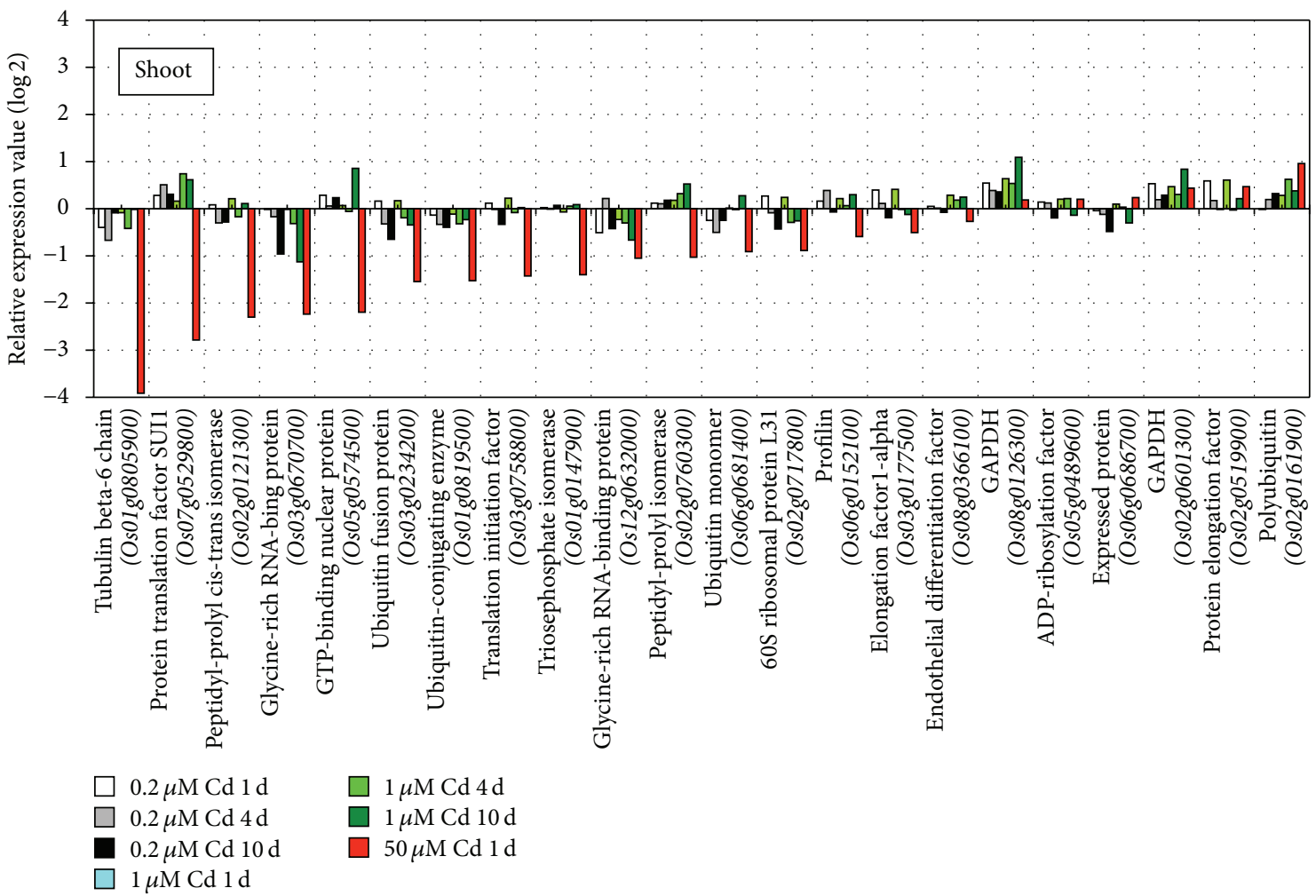

(b)

FIGURE 3: Response of constitutively expressed genes in roots and shoots to Cd exposure. The relative expression of constitutively expressed genes [27] in roots (a) and shoots (b) is shown under Cd exposure at each stress time point (1,4, and $10 \mathrm{~d}$ ) during $0.2 \mu \mathrm{M}$ (white, grey, and black) and $1 \mu \mathrm{M}$ (light blue, light green, and green) Cd exposure compared with nontreatment $(0 \mathrm{~d})$. The red bar shows the relative expression at $1 \mathrm{~d}$ under $50 \mu \mathrm{M}$ Cd exposure. The $x$-axis shows the genes and the $y$-axis shows relative expression. Wang et al. [27] suggested the following genes as candidates for constitutive expression: glycine-rich RNA-binding protein (Os12g0632000), expressed protein (Os06g0686700), profilin (Os06g0152100), ADP-ribosylation factor (Os05g0489600), triosephosphate isomerase (Os01g0147900), glycine-rich RNA-binding protein (Os03g0670700), peptidyl-prolyl cis-trans isomerase (Os02g0121300), endothelial differentiation factor (Os08g0366100), ubiquitin monomer (Os06g0681400), protein translation factor SUI1 (Os07g0529800), GAPDH (Os08g0126300), polyubiquitin (Os02g0161900), protein elongation factor (Os02g0519900), translation initiation factor (Os03g0758800), ubiquitin-conjugating enzyme (Os01g0819500), GTP-binding nuclear protein (Os05g0574500), peptidyl-prolyl isomerase (Os02g0760300), and 60S ribosomal protein L31 (Os02g0717800). Their paper also introduced the following genes that have frequently been used as internal controls in expression analyses: elongation factorl-alpha (Os03g0177500), ubiquitin fusion protein (Os03g0234200), GAPDH (Os02g0601300), and tubulin beta-6 chain (Os01g0805900). 


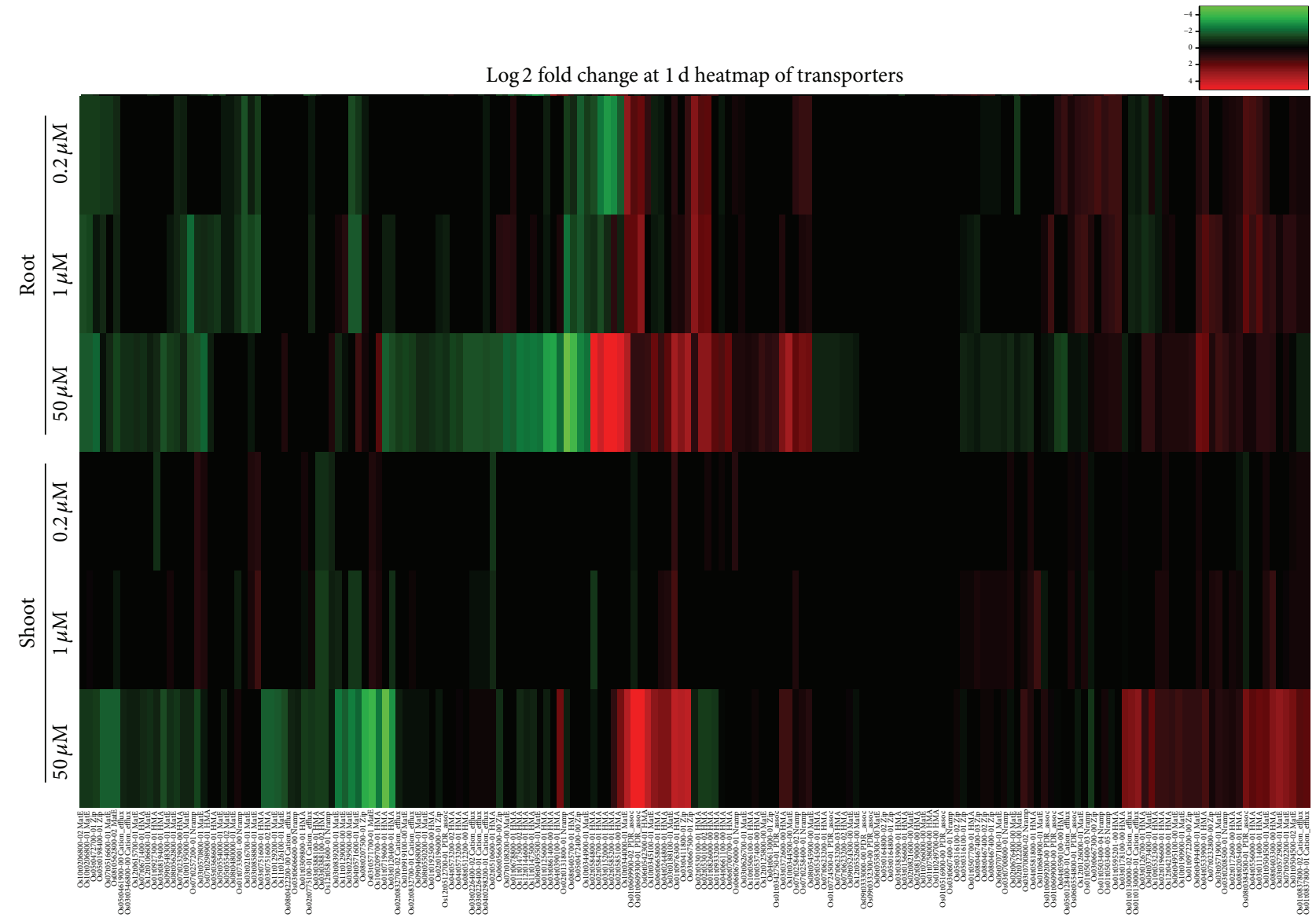

FIGURE 4: Expression profiling of metal ion transporter genes in roots and shoots under Cd exposure at $1 \mathrm{~d}$ demonstrates Cd concentrationdependent differences. Heatmap analysis of metal ion transporters containing Pfam domains [PF01554 (MatE), PF08370 (PDR_assoc), PF01545 (Cation_efflux), PF02535 (Zip), PF00403 (HMA), and PF01566 (Nramp)]. The relative expression values under 0.2, 1, and 50 $\mu$ M $\mathrm{Cd}$ (data from [4]) are presented. The color scale shows log2-transformed transcript levels for each gene.

\section{Conflict of Interests}

The authors declare that there is no conflict of interests regarding the publication of this paper.

\section{Authors' Contribution}

Youko Oono and Takashi Matsumoto conceived and designed the experiments. Takashi Matsumoto performed sampling. Hiroyuki Kanamori, Harumi Sasaki, and Satomi Mori performed the experiments. Youko Oono, Takayuki Yazawa, and Hiroyuki Kanamori analyzed the data and contributed analysis tools. Youko Oono wrote the paper. Hirokazu Handa and Takashi Matsumoto contributed valuable insights into the discussion and revision of the paper. Youko Oono and Takayuki Yazawa contributed equally to this work.

\section{Acknowledgments}

The authors thank Ms. F. Aota, Ms. K. Ohtsu, and Ms. K. Yamada for technical assistance. This work was supported by a grant from the Ministry of Agriculture, Forestry and Fisheries of Japan (Genomics for Agricultural Innovation, RTR-0001).

\section{References}

[1] CODEX, "Report of the 38th session of the CODEX Committee on Food Additives and Contaminants," ALINORM 06/29/12, Codex Alimentarius Commission, 2006.

[2] B. Halliwell and J. M. C. Gutteridge, "Oxygen-toxicity, oxygen radicals, transition-metals and disease," Biochemical Journal, vol. 219, no. 1, pp. 1-14, 1984.

[3] Z. Wang, M. Gerstein, and M. Snyder, "RNA-Seq: a revolutionary tool for transcriptomics," Nature Reviews Genetics, vol. 10, no. 1, pp. 57-63, 2009.

[4] Y. Oono, T. Yazawa, Y. Kawahara et al., "Genome-wide transcriptome analysis reveals that cadmium stress signaling controls the expression of genes in drought stress signal pathways in rice," PLoS ONE, vol. 9, no. 5, Article ID e96946, 2014.

[5] S. Yoshida, D. A. Forno, J. H. Cock, and K. A. Gomez, Laboratory Manual for Physiological Studies of Rice, International Rice Research Institute, Manila, Philippines, 3rd edition, 1976. 
[6] S. Sauve, W. A. Norvell, M. McBride, and W. Hendershot, "Speciation and complexation of cadmium in extracted soil solutions," Environmental Science \& Technology, vol. 34, no. 2, pp. 291-296, 2000

[7] Y. Kawahara, Y. Oono, H. Wakimoto et al., "TENOR: database for comprehensive mRNA-Seq experiments in rice," Plant and Cell Physiology, vol. 57, no. 1, article e7, 2016.

[8] M. Martin, "Cutadapt removes adapter sequences from highthroughput sequencing reads," EMBnet Journal, vol. 17, no. 1, pp. 10-12, 2011.

[9] Y. Oono, Y. Kawahara, H. Kanamori et al., "mRNA-seq reveals a comprehensive transcriptome profile of rice under phosphate stress," Rice, vol. 4, no. 2, pp. 50-65, 2011.

[10] H. Li and R. Durbin, "Fast and accurate short read alignment with Burrows-Wheeler transform," Bioinformatics, vol. 25, no. 14, pp. 1754-1760, 2009.

[11] A. Mortazavi, B. A. Williams, K. McCue, L. Schaeffer, and B. Wold, "Mapping and quantifying mammalian transcriptomes by RNA-Seq," Nature Methods, vol. 5, no. 7, pp. 621-628, 2008.

[12] M. Zhang, X. Liu, L. Yuan et al., "Transcriptional profiling in cadmium-treated rice seedling roots using suppressive subtractive hybridization," Plant Physiology and Biochemistry, vol. 50, no. 1, pp. 79-86, 2012.

[13] K. Lee, D. W. Bae, S. H. Kim et al., "Comparative proteomic analysis of the short-term responses of rice roots and leaves to cadmium," The Journal of Plant Physiology, vol. 167, no. 3, pp. 161-168, 2010.

[14] K. Shah, R. G. Kumar, S. Verma, and R. S. Dubey, "Effect of cadmium on lipid peroxidation, superoxide anion generation and activities of antioxidant enzymes in growing rice seedlings," Plant Science, vol. 161, no. 6, pp. 1135-1144, 2001.

[15] L. Perfus-Barbeoch, N. Leonhardt, A. Vavasseur, and C. Forestier, "Heavy metal toxicity: cadmium permeates through calcium channels and disturbs the plant water status," Plant Journal, vol. 32, no. 4, pp. 539-548, 2002.

[16] R. Mittler, S. Vanderauwera, N. Suzuki et al., "ROS signaling: the new wave?" Trends in Plant Science, vol. 16, no. 6, pp. 300-309, 2011.

[17] C. Frova, "The plant glutathione transferase gene family: genomic structure, functions, expression and evolution," Physiologia Plantarum, vol. 119, no. 4, pp. 469-479, 2003.

[18] C. Cosio and C. Dunand, "Specific functions of individual class III peroxidase genes," Journal of Experimental Botany, vol. 60, no. 2, pp. 391-408, 2009.

[19] C. Cobbett and P. Goldsbrough, "Phytochelatins and metallothioneins: roles in heavy metal detoxification and homeostasis," Annual Review of Plant Biology, vol. 53, pp. 159-182, 2002.

[20] K. Yamaguchi-Shinozaki and K. Shinozaki, "Transcriptional regulatory networks in cellular responses and tolerance to dehydration and cold stresses," Annual Review of Plant Biology, vol. 57, pp. 781-803, 2006.

[21] R. Cailliatte, A. Schikora, J.-F. Briat, S. Mari, and C. Curie, "High-affinity manganese uptake by the metal transporter NRAMP1 is essential for Arabidopsis growth in low manganese conditions," Plant Cell, vol. 22, no. 3, pp. 904-917, 2010.

[22] A. Sasaki, N. Yamaji, K. Yokosho, and J. F. Ma, "Nramp5 is a major transporter responsible for manganese and cadmium uptake in rice," Plant Cell, vol. 24, no. 5, pp. 2155-2167, 2012.

[23] N. Satoh-Nagasawa, M. Mori, N. Nakazawa et al., "Mutations in rice (Oryza sativa) heavy metal ATPase 2(OsHMA2) restrict the translocation of zinc and cadmium," Plant and Cell Physiology, vol. 53, no. 1, pp. 213-224, 2012.
[24] Y. O. Korshunova, D. Eide, W. G. Clark, M. L. Guerinot, and H. B. Pakrasi, "The IRT1 protein from Arabidopsis thaliana is a metal transporter with a broad substrate range," Plant Molecular Biology, vol. 40, no. 1, pp. 37-44, 1999.

[25] N. E. Grossoehme, S. Akilesh, M. L. Guerinot, and D. E. Wilcox, "Metal-binding thermodynamics of the histidine-rich sequence from the metal-transport protein IRT1 of Arabidopsis thaliana," Inorganic Chemistry, vol. 45, no. 21, pp. 8500-8508, 2006.

[26] S. Lee and G. An, "Over-expression of OsIRT1 leads to increased iron and zinc accumulations in rice," Plant, Cell and Environment, vol. 32, no. 4, pp. 408-416, 2009.

[27] L. Wang, W. Xie, Y. Chen et al., "A dynamic gene expression atlas covering the entire life cycle of rice," Plant Journal, vol. 61, no. 5, pp. 752-766, 2010.

[28] G. M. He, X. P. Zhu, A. A. Elling et al., "Global epigenetic and transcriptional trends among two rice subspecies and their reciprocal hybrids," Plant Cell, vol. 22, no. 1, pp. 17-33, 2010.

[29] T. T. Lu, G. J. Lu, D. L. Fan et al., "Function annotation of the rice transcriptome at single-nucleotide resolution by RNA-seq," Genome Research, vol. 20, no. 9, pp. 1238-1249, 2010.

[30] P. Pedas, C. K. Ytting, A. T. Fuglsang, T. P. Jahn, J. K. Schjoerring, and S. Husted, "Manganese efficiency in Barley: identification and characterization of the metal ion transporter HvIRT1," Plant Physiology, vol. 148, no. 1, pp. 455-466, 2008. 

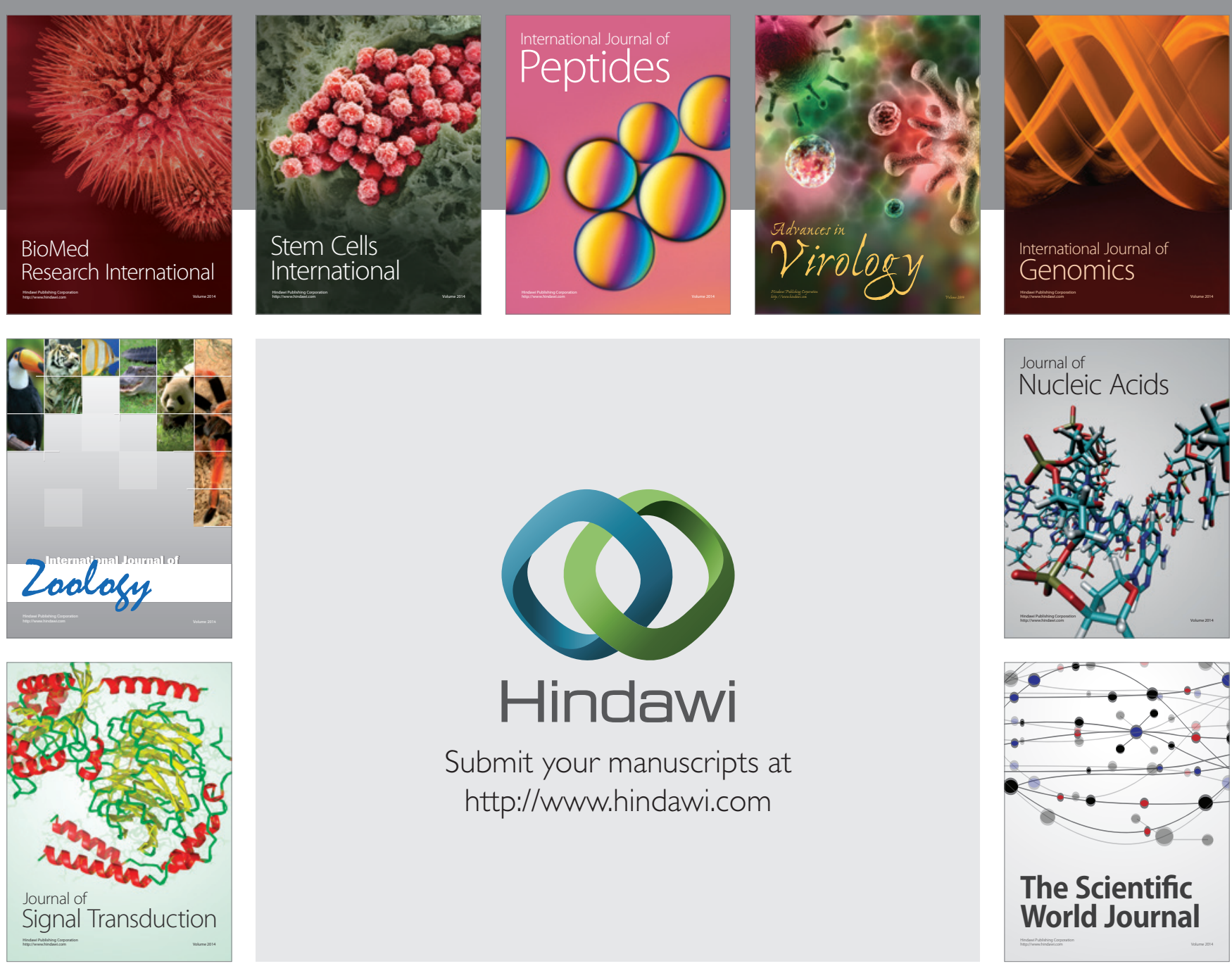

Submit your manuscripts at

http://www.hindawi.com
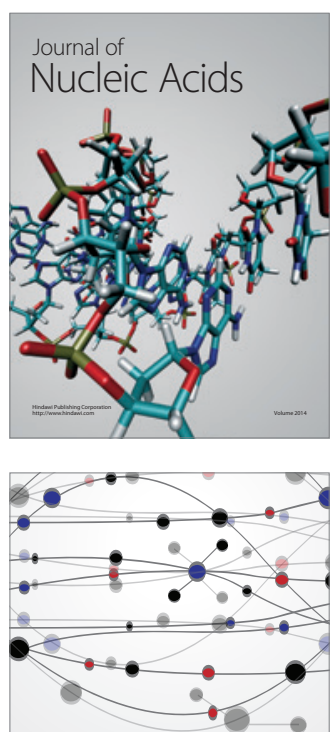

The Scientific World Journal
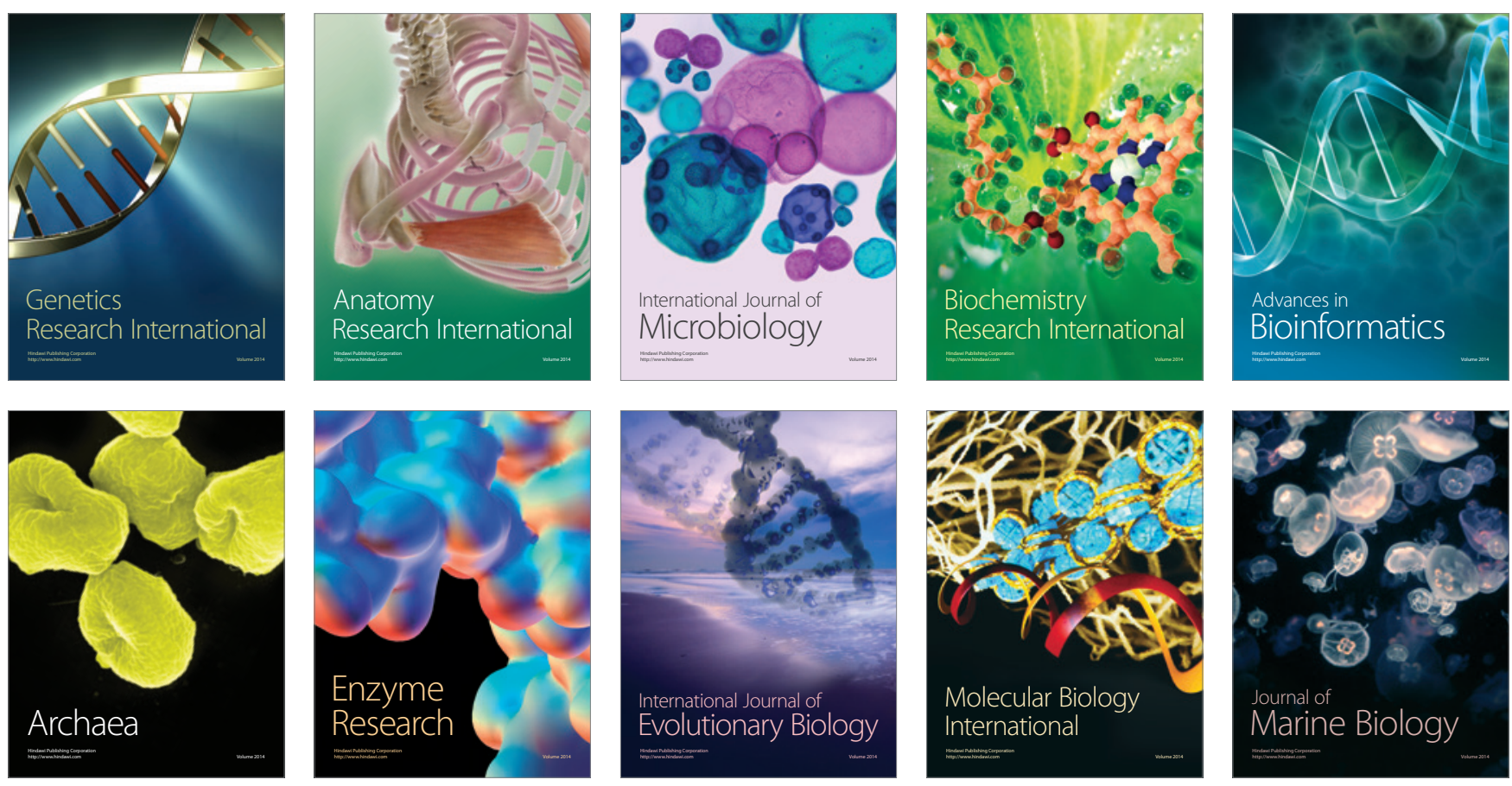